\title{
ALGUNAS CONSIDERACIONES HISTÓRICAS, EPISTEMOLÓGICAS Y DIDÁCTICAS PARA EL ABORDAJE DE LATEORÍA DE LA RELATIVIDAD ESPECIAL EN EL NIVEL MEDIO Y POLIMODAL
}

Irene Arriassecq ${ }^{1}$

lleana M aría Greca²

\begin{abstract}
Resumen: En este trabajo se presentan una serie de consideraciones, emergentes de aportes recientes de la H istoria de la Ciencia, la Filosofía de la Ciencia y la D idáctica de las C iencias, relevantes para la introducción de la Teoría Especial de la Relatividad (TER) en la enseñanza secundaria. Se considera que los aspectos destacados deberían estar presentes en los libros de texto utilizados, por lo que los ejes propuestos pueden servir de base para el análisis de las obras didácticas que abordan el tema de la teoría especial de la Relatividad (TER). El trabajo pretende, además, servir de aporte para los docentes, presentando la TER contextualizada desde el punto de vista histórico, epistemológico y didáctico.
\end{abstract}

Unitérminos: H istoria y Filosofía de la Ciencia; Enseñanza de la Física; Análisis de libros de texto, Teoría Especial de la Relatividad.

Abstract: A series of considerations, emergent of recent contributions of the History of the Science, the Philosophy of the Science and the D idactics of the Sciences, important for the introduction of the Special Theory of the Relativity (TER) in the secondary education are presented in this report. Considering that the relevant aspects of this subject should be attended in the used textbooks, our proposed axes can serve as base for the analysis of the didactic works that approach the topic of the special theory of the Relativity (TER). With this work we hope to make a contribution for teachers, contextualizing theTER from the his torical, epistemological and didactic point of view.

Keywords: H istory and Philosophy of Science; Physics teaching; Analysis of textbooks, Special Relativity Theory.

\section{Introducción}

$\mathrm{H}$ oy en día existe consenso entre docentes e investigadores en la didáctica de las Ciencias respecto a que los grandes logros de la ciencia de este siglo, y de la Física en particular, deberían formar parte de la actualización de la currícula escolar (H olcomb, 1997; Villani e Arruda, 1998). D entro de los diversos temas posibles de ser abordados dentro de esta temática parecen existir algunos que resultan particularmente interesantes para los alumnos. Este parece ser el caso de la Teoría de la Relatividad (TER). Según los resultados

\footnotetext{
1 Professora da UNCPBA - Facultad de Ciencias. Exactas - Departamento de Formación Docente, Campus Universitario, Paraje Arroyo Seco, Argentina. Programa Internacional de Doctorado en Enseñanza de las Ciencias Universidad de Burgos, España y Universidade Federal do Rio Grande do Sul, Brasil (e-mail: irenearr@exa.unicen.edu.ar).

2 Professora do D epartamento de M atemática e do Programa de Pós-Graduação em Ensino de Ciências e M atemática, U niversidade Luterana do Brasil. Campus Canoas, Rio Grande do Sul, Brasil. Programa Internacional de D outorado em Ensino das Ciências. U nivers dade de Burgos, Espanha eU niversidade Federal do Rio G rande do Sul, Brasil. (email: ilegreca@terra.com.br).
} 
de una investigación que analizó diversos aspectos de la incorporación de la Física Contemporánea en la enseñanza de la escuela media y Polimodal ${ }^{4}$ en más del cincuenta por ciento de las provincias argentinas, fue posible observar que la teoría más conocida por los estudiantes como referente de la Física, y la que más les interesaría aprender, era justamente la teoría de la Relatividad (C apuano et al., 1997). LaTER, por otra parte, se presenta en los planes ministeriales de la Argentina como tema en Física de segundo año de los Polimodales con orientación en C S. N aturales: ".... Se propone el estudio de las leyes de conservación y algunas cuestiones centrales de la física del siglo XX. Por ejemplo, la M ecánica Cuántica y la Teoría de la Relatividad, contenidos que posibilitan la discusión de los procesos que definen las grandes transformaciones en el pensamiento científico..." (D ocumento del Consejo General de cultura y Educación de la Pcia. de Bs. As., 1999).

D esde el punto de vista de la enseñanza de las ciencias, la TER es un tema particularmente rico dado que los primeros contactos de los alumnos con el mismo implican, 0 deberían implicar, un verdadero punto de inflexión en el conocimiento de la Física, pues lo que puede haber de continuidad entre la física clásica y la relativista es menos relevante que aquello que las diferencia. Por eso, parecería necesario que, en relación con laTER, los alumnos consiguiesen una evolución conceptual entendida en sentido toulminiano (Toulmin, 1977), o sea, no como un cambio conceptual en sentido literal - reemplazo de una concepción existente por otra adquirida mediante la instrucción formal - sino como coexistencia de ambas concepciones, siendo lo deseable que el alumno fuese capaz de identificar el campo de validez de la "vieja" concepción y aceptase la plausibilidad y eficiencia de la "nueva". Sin embargo, las investigaciones real izadas sobre la comprensión conceptual de la TER muestran que esta evolución conceptual es difícil de ser conseguida y esta dificultad aparece tanto en los alumnos como en los propios docentes (entre otros, Aleman Berenguer, R.,1997, Aleman Berenger, R. y Pérez Selles, J., 2000, Pietrocola e Zylbersztajn, 1999, Villani 1998).

Aunque estos elementos muestren la importancia de la introducción de la TER en el ciclo medio, aún falta saber cómo hacerlo. Algunas propuestas a este respecto han aparecido en los últimos años (por ejemplo, G.I.R.E.P., 1991; Bertali et al., 1979, Solbes, 1986 y Borghi et al. 1993 en Villani y Arruda, 1998), siendo divergentes los resultados de estas implementaciones. Por un lado, al gunos resultados de investigaciones parecerían indicar que el "entusiasmo" por aprender la TER permitiría sortear las dificultades que esta presenta. 0 tros trabajos, por el contrario, muestran un panorama bien diferente: los estudiantes interpretarían los conceptos relativistas desde su sistema de creencias espontaneas y no a partir de los nuevos conceptos en el marco de la TER (Villani y Arruda, 1998). Al respecto, Villani

\footnotetext{
${ }^{3}$ D enominaremos como Física Contemporánea a la Física desarrollada durante el siglo XX, siguiendo el uso corriente en la Filosofía de la C iencia. En esteámbito, se aplica la denominación de Física M oderna a la física desarrollada durante la Edad M oderna (siglos XVII e XVIII), aunque cabe aclarar que no existe consenso al respecto. Así, por ejemplo, algunos autores adoptan el criterio de usar "ciencia moderna" para la Física desarrollada en la Edad M oderna (S. XVII), y "Física M oderna" para a M ecánica Cuántica, en contraposición a la idea de Física Clásica.

${ }^{4}$ En 1992 se pone en marcha el proceso de reforma educativa en la República Argentina, mediante la promulgación de la Ley Federal de Educación y los acuerdos entre el gobierno nacional y los gobiernos provinciales, en el seno del Consejo Federal deCultura y Educación. El proceso de transformación de la educación, se caracteriza, entre otros aspectos, por la transferencia de los servicios educativos desde el gobierno nacional a las provincias (salvo el nivel universita rio), mediante la propuesta de una nueva organización de los estudios, pasando de una estructura que diferenciaba el nivel primario (siete años de escolaridad) del secundario (cinco años de escolaridad) a otra en la que la educación gene ral básica (EGB) se extiende a 9 años a los que se agregan tres años de escolaridad secundaria denominado polimodal y que reemplaza al ciclo superior de educación media. Esta transformación en la educación propuso como objetivos para el nivel polimodal profundizar los contenidos adquiridos en la EGB y ofrecer la capacitación en grandes campos del conocimiento y del quehacer social y productivo.
} 
(1992), por ejemplo, sostiene que la aceptación de la TER por parte de los estudiantes es sólo provisional, ya que es contraria a sus convicciones profundas.

En la búsqueda de como incorporar estos contenidos en la enseñanza de nivel medio (Polimodal), un aspecto que aun no ha sido estudiado, y que consideramos importante, es el del análisis de los libros de texto que utilizan tanto docentes como alumnos, dado que la efectiva incorporación curricular está fuertemente influenciada por los contenidos que aparecen en los libros didácticos que los profesores utilizan como referencia. Como se sabe, los docentes, y también los alumnos, tienden a utilizar los libros de texto como uno de los principales recursos en el proceso de enseñanza - aprendizaje: "el libro escolar impregna las prácticas, transmite contenido, organiza las experiencias de los alumnos, establece tipos de interacción, y todo eso lo hace con una enorme fuerza legal" (Litwin,1997). De esta forma, asumiendo que el docente, además de elegir los textos que él mismo consulta, condiciona fuertemente los que utiliza el alumno, acordamos con Islas y Stipcich (2000) cuando sugieren al docente dos ejes para el análisis de los textos: uno vinculado con qué cosas contienen los mismos y otro con cómo estructura el contenido. Según ese trabajo, dentro del tópico de qué contienen, deberían contemplarse los contenidos disciplinares específicos, las actividades en relación con los contenidos y los tópicos vinculados con la historia y la filosofía de la ciencia. El trabajo que aquí presentamos se centra, principalmente, en la discusión de elementos que deberían ser llevados en consideración en relación con el último de los ítems, dado el creciente consenso entre los investigadores en el área de la didáctica de las ciencias acerca de la importancia de la introducción de tópicos de Física desde un enfoque histórico e epistemológico moderno.

Partimos del presupuesto que la historia de la ciencia debería utilizarse en los textos y en el aula con varios objetivos, entre ellos (H odson, 1986 y K ragh, 1989):

que los alumnos comprendan las dificultades, los obstáculos que se debieron superar y los contextos culturales, filosóficos, tecnológicos, etc., distintos del actual; en que se produjeron las teorías científicas;

que la ciencia es una actividad humana y está realizada por hombres que aportan contribuciones parciales respondiendo preguntas que se plantean en cada época, o sea, que rara vez hay "un sólo descubridor";

que los científicos, en cada momento histórico, no pensaban en nuestros "términos actuales", ya que utilizaban las herramientas lógicas, metodológicas, epistemológicas y las tradiciones predominantes de su medio y su época.

En suma, el uso de la historia de la ciencia debería mostrar que el conocimiento actual es el resultado de un largo proceso, en donde la interrelación teoría - empiria es constante y sobre el cual los factores filosóficos, culturales, sociales e incluso estéticos, tienen un peso importante. Sin embargo, es relevante aclarar que el uso de la historia de ciencia como recurso didáctico para estructurar la presentación de un tema complejo, tanto en libros de texto como en clase, no debería derivar en simplificaciones extremas que distorsionen el sentido de la historia de la ciencia, y de la propia ciencia, en la enseñanza (M atthews, 1994 y Lombardi, 1997).

D esde la perspectiva planteada, intentamos determinar algunos ejes (la discusión de cada eje es brevemente ilustrada con al gunos ejemplos) que consideramos necesarios para la introducción de laTER en la enseñanza secundaria, ejes estos que deberían plasmarse en los textos didácticos. (D ejamos para un próximo artículo el análisis propiamente dicho a

\footnotetext{
${ }^{5} \mathrm{~N}$ o se excluye que no los haya, pero estos son casos aislados en el pasado (Boido, comunicación personal).
} 
partir de estos ejes de los libros didácticos más utilizados en el nivel de enseñanza secundaria y universitaria.)

\section{Polimodal}

\section{Propuesta de algunos ejes estructuradores para el abordaje de la TER a nivel}

\section{- Contextualización histórica de la TER}

Una adecuada contextualización histórica del surgimiento de laTER debería contemplar aspectos tales como un panorama del estado de la Física en la época en que surge la teoría y las contribuciones de investigadores que prepararon el terreno para laTER. En ese sentido podría discutirse el estado de la Física hasta 1905. Un aspecto interesante en relación con esta cuestión es la caracterización del Programa M ecanicista newtoniano. H asta finales del siglo XIX se aceptaba que el constituyente de la realidad física era materia, discreta o continua, que era influida por fuerzas (de contacto 0 a distancia) de acuerdo con las leyes de la M ecánica. D esde de esta pers pectiva, el universo era considerado como una gran máquina, creada por un "Dios Ingeniero", infinita y corpuscular, sin lugares privilegiados, donde los corpúsculos se movían en el vacío. ${ }^{6}$ A partir de la cosmovisión newtoniana, los sucesores de N ewton durante casi doscientos años, tuvieron la convicción de disponer de un marco teórico adecuado para explicar "todos" los fenómenos naturales: ópticos, calóricos, magnéticos, eléctricos, químicos y hasta biológicos. En síntesis: "EI mecanicismo, a su vez, adquirió una significación precisa: esla tesis según la cual todo fenómeno observable puede ser explicado por medio de la mecánica newtoniana aplicada a los corpúsculos que cons tituyen el universo, únicas entidades reales que habitan el espacio y el tiempo" (Boido, 1996, p. 357).

En el último tercio del siglo XIX se hizo manifiesta la incompatibilidad del Programa M ecanicista con la Física del momento, en particular con la física relacionada a los fenómenos electromagnéticos, siendo varios los científicos que realizaron aportes para una nueva visión de la naturaleza. Faraday, por ejemplo, propuso la tesis que la única entidad físca real es la fuerza. Para él, el mundo seria un vasto campo defuerzas, donde unas fuerzas actuarían sobre otras fuerzas contiguas, o sea, no a distancia, y donde las partículas serían los puntos de convergencia de fuerzas de gran intensidad. Como no existe la "acción a distancia", toda perturbación del campo en un punto del espacio debería comunicarse a los puntos vecinos y como se demora un cierto tiempo en realizar este proceso, la perturbación se propagaría con velocidad infinita. A partir de este esquema, que se enfrenta a las ideas mecanicistas newtonianas, Faraday consigue explicar varios fenómenos eléctricos.

O tro aporte importante fue el de M axwell, quien sintetizó los resultados de décadas de experimentación en fenómenos eléctricos y magnéticos en una teoría unificada de electricidad y magnetismo, condensada en cuatro ecuaciones que permiten demostrar que la interacción de los fenómenos eléctricos con los magnéticos puede crear un movimiento perpetuo de electricidad y magnetismo en forma de ondas, que se propagaban a la misma velocidad que la luz. D ado que $M$ axwell estaba dentro del contexto mecanicista, estas ondas debían propagarse en un medio material, un medio corpóreo, para que sus vibraciones pudiesen "sostener" las fuerzas electromagnéticas de la teoria. Este medio hipotético posteriormente fue denominado "éter

\footnotetext{
${ }^{6}$ Esta visión del programa mecanicista newtoniano no era compartida por la otra rama del mecanicismo, el cartesianismo: en la visión de universo aceptada por los científicos continentales se asumía un plenum sin vacío.

${ }^{7}$ Es interesante clarificar la idea de que $M$ axwell habría reeditado la noción de éter. En realidad, como ya indicamos antes, la idea de no-existencia de vacío no había desaparecido para la rama cartesiana del programa mecanicista. Lo que M axwell hace es introducir esta idea en la rama newtoniana, en un esfuerzo para salvar el mecanicismo de su teoría (Flichman, comunicación personal).
} 
electromagnético", y su búsqueda se convirtió en un tema central de investigación. De hecho, los sucesivos fracasos para la detección del éter generaron una serie de trabajos para reformar el programa mecanicista, sin una revisión profunda de sus fundamentos. Estas tentativas de reforma fueron encabezadas fundamentalmente por Lorentz, cuyos trabajos sirven de base para la elaboración de la TER. 0 tro aspecto fundamental de la teoría electromagnética propuesta por $M$ axwell, en relación con la TER, se refiere a que de sus ecuaciones es posible deducir que para un observador en el éter las ondas se propagan siempre a la misma velocidad, con independencia de la velocidad de la fuente de esas ondas con respecto al observador, lo que está en contradicción con los postulados del programa mecanicista newtoniano.

En el origen de la TER es posible rastrear también la influencia más "epistemológica" debida a M ach, a través de sus críticas a la M ecánica newtoniana. Según H olton (1982), esta influencia se manifiesta en dos aspectos cruciales en el artículo de Einstein de 1905 sobre relatividad. U no de ellos es la insistencia de Einstein, desde el comienzo de su artículo, en que los problemas fundamentales de la física no pueden resolverse sin un profundo análisis epistemológico, particularmente acerca del significado de nociones como espacio y tiempo. El otro aspecto donde se manifestaría la influencia de $M$ ach se relaciona con la identificación que hace Einstein de la realidad con lo que se percibe a través de las "sensaciones". (Existe abundante correspondencia entre M ach y Einstein donde se puede analizar en profundidad hasta que punto, inicialmente, Einstein habría recibido fuerte influencia del pensamiento de $M$ ach.)

D entro de este contexto, en que la vieja cosmovisión era colocada en jaque en relación con un número importante de problemas de diversos orígenes (empíricos, teóriCOS, epistemológicos) surge la TER, brindando explicaciones que podrían solucionar parte de estos problemas, aunque con el costo de cambiar de cosmovisión.

\section{- Reflexión epistemológica referida a la génesis de la TER}

D esde el punto de vista epistemológico, existen varios aspectos, presentes en los debates epistemológicos actuales, cuya discusión puede ser enriquecedora. Por ejemplo, las reflexiones acerca de la génesis de una teoría, sus contrastaciones empíricas, las aplicaciones de la misma, el rol de la comunidad científica en el desarrollo de una teoría y las influencias de la producción científica en la sociedad.

\section{Rol de la experimentación en la génesis de la TER}

Existen numerosos trabajos del área de historia de la ciencia en que detenidos análisis de documentos, tanto científicos como generales, incluyendo cartas, reportajes, declaraciones y notas autobiográficas, permiten afirmar que el rol del experimento de $M$ ichelson en la génesis de la TER parecería haber sido menor e indirecto.

Esta opinión, sin embargo, fue corriente entre los científicos. D e hecho, la opinión del ambiente científico hasta hace poco tiempo atrás, era considerar la TER como un gran éxito teórico, la respuesta finalmente correcta al experimento de $\mathrm{M}$ ichelson y su continuación natural (Villani, 1981). Esta opinión, marcada por la visión empirista de los propios científicos, pasó a los libros de enseñanza y de divulgación, a tal punto que ni los excelentes libros del curso Física de Berkeley y las Feynman's Lectures on Physics escapan a esta visión. Holton (1973) coloca que varios acontecimientos coayudaron a afianzar esta opinión: el clima histórico particular, dada la difícil aceptación tanto de los resultados de la experiencia de M ichelson como de la TER; las propias manifestaciones de Einstein en sus 
primeras publicaciones didácticas; las finalidades de los libros didácticos: convencer a los estudiantes de la bondad de una teoría estableciendo relaciones entre teoría y experimentación y la propia racionalidad experimentalista que dominaba el ambiente científico de la época.

A pesar de esto, una revisión cuidadosa de la historia del surgimiento de la TER permite especular que aunque el experimento de $M$ ichelson no se hubiera realizado, no hubiera influido en el surgimiento de la TER: antes de 1905 Einstein, a través del trabajo de Lorentz, conocía de varios intentos fallidos para determinar movimientos de la Tierra respecto del éter (H olton, 1982). M ás aún, $\mathrm{H}$ olton indica que el interés de Einstein surge de una insatisfacción de tipo estético y no de una preocupación de dar respuesta a resultados experimentales contradictorios. 0 sea, el punto de partida de la TER no se trataría de un desacuerdo entre teoría e experimento sino de la necesidad de crear un punto de vista nuevo para evitar la asimetría en la explicación de los efectos del movimiento entre un imán y un conductor.

Insistir en presentaciones de la TER donde se sugiere que la experiencia de $M$ ichelson es el punto de partida para que Einstein desarrolle su teoría, contribuye a generar en los alumnos una visión distorsionada de la actividad científica, fomentando una visión radicalmente empirista de la ciencia, lo que no es deseable, dado que, desde el punto de vista epistemológico, actualmente existen planteos superadores (M atthews, 1994).

\section{O riginalidad de la TER}

La originalidad del trabajo de Einstein ha suscitado una vasta controversia (ver, a este respecto, una presentación muy interesante en los trabajos de Villani, 1981-1985, donde este autor presenta también por que el programa de Einstein triunfa con relación al de Lorentz, aunque no existían, en la época, evidencias empíricas superiores a favor de las teorías de uno o del otro.). D e hecho existen hoy tanto argumentos a favor de la originalidad de su teoría, como otros que sostienen que es el corolario lógico de trabajos de otros científicos. Esto es, en nuestra opinión, una posibilidad más de rescatar en el aula aspectos epistemológicos cuya discusión puede influir favorablemente en la visión de ciencia que los alumnos van construyendo a lo largo del proceso de aprendizaje de la misma. Independientemente de hasta donde Einstein fue influenciado por otros científicos, conocer teorías que se ocuparon de problemas en común con los tratados por la TER, puede posibilitar la comprensión de la dimensión revolucionaria de la misma. Además, cabe aclarar que entre los historiadores no existe completo acuerdo respecto de cuáles eran los trabajos que realmente conocía Einstein con anterioridad a la publicación de su teoría.

Para dar un ejemplo al respecto tomemos el caso de dos opiniones: la de Sir Edmund W hittaker, destacado físico teórico que se dedicó a la H istoria de la Ciencia (cabe aclarar que W hittaker no es considerado por la comunidad de historiadores de la ciencia como un "historiador de la ciencia", sino como un físico que opinó acerca de al gunas cuestiones vinculadas a la historia de la física) y fue testigo de la revolución que provocó la TER y la de G erald H olton, profesor de Física de $\mathrm{H}$ arvard, físico e historiador de la ciencia. Se escogen estas dos perspectivas porque más allá del reconocido prestigio de quienes las sustentan, plantean posturas contrapuestas que posibilitan un análisis crítico del surgimiento de la TER.

Para W hittaker el artículo de Einstein de 1905 es "... en el que se exponía, con algunas ampliaciones, la teoría de la relatividad de Poincaré y Lorentz... " (Whittaker en 
H olton - en P. Williams comp. -, 1995). ${ }^{8}$ Respecto al artículo de Lorentz, publicado también en 1904, Wittaker plantea que es el mayor aporte para la TER, asumiendo que Einstein utilizó las transformaciones de Lorentz. Sin embargo Holton (en P. Williams comp., 1995) afirma que tanto Einstein como personas de su entorno lo negaron reiteradamente; incluso el propio Einstein afirmó no haber leído dicho artículo. Como afirma Holton (ibid.), "... el estudio detallado de la situación histórica es, en mi opinión, un primer paso importante en aquellas discusiones que pretenden basar las consideraciones epistemológicas en casos "reales". Lo cual no siempre es fácil...".

C on este ejemplo pretendemos ilustrar que, desde una postura de la enseñanza de la ciencia histórica y epistemológicamente contextualizada, sería deseable no presentar la TER como la invención de un científico genial, sino mostrar la existencia de otros científicos que en la época trabajaban en los mismos problemas, o sea, enfatizar la discusión con los alumnos en relación con que ninguna teoría científica "surge" en un vacío conceptual.

Además de estos puntos, quizás seria interesante analizar con los alumnos el tipo de explicación que laTER instauró en el ambiente científico. Battimelli (en Villani, 1981, p. 30) considera que la gran novedad de la teoría einsteniana está en el hecho en que ofrece un nuevo modelo de aquello que debe ser entendido por una explicación científica y, por lo tanto, por una teoría en Física, modelo netamente teórico, basado en algunos principios fundamentales. Este tipo de paradigma de explicación invierte, según Battimelli, "el enfoque que era habitual en la época, en vez de reconstruir propiedades de los fenómenos a partir de la interacción de los componentes fundamentales, establece como punto de partida de la teoría las propiedades empíricamente observadas de los fenómenos, generalizadas a principios de validad universal."

\section{Referencia a comprobaciones experimentales de la TER}

Sin lugar a dudas las contrastaciones empíricas en la Física cumplen un rol decisivo en algunos casos, sin que con esta afirmación se deje de reconocer que existen discusiones epistemológicas en torno a lo definitivo o no de una contrastación exitosa o fallida. Popper, Kuhn, Lakatos y Feyerabend, por mencionar sólo algunos de los epistemólogos más conocidos, no coinciden en relación con esta cuestión. En el caso de la TER, no han sido menores los esfuerzos por ponerla a prueba y dados los aspectos particularmente contraintuitivos que posee la teoría para quienes la abordan por primera vez, no debería obviarse en los textos y en clase la mención de las distintas corroboraciones de la misma. A modo de ejemplo de este tipo de publicaciones se pueden mencionar un artículo de Rossi y Hall de 1941 en Physical Review y otro de Friech y Smith en American Journal of Physics, donde se presentan experiencias que comprueban que el ritmo de pulsación de un reloj, para un observador en reposo, no coincide con el que mide un observador en movimiento (M ook, D. y Vargish, T., 1998).

Sin embargo, seria necesario resaltar que estas confirmaciones experimetales son bien posteriores al contexto de aceptación de la TER. En la época, la TER no presentaba

\footnotetext{
${ }^{8}$ Brevemente, el artículo de Poincaré de 1904, al que se refiere Wittaker consiste en un análisis de las críticas que desde la física de la época se realizaron a los principios clásicos, incluyendo el principio de relatividad de Galileo y N ewton. Poincaré pretendía argumentar acerca de la necesidad de un nuevo desarrollo de la física, "O quizás debamos construir toda una nueva mecánica que hasta ahora sólo hemos logrado entrever y en la que, al aumentar la inercia con la velocidad, la velocidad de la luz se convertirá en un límite infranqueable" (H olton, 1969), pero no enuncia el nuevo principio de relatividad, ni desarrolla su propuesta.
} 
superioridad empírica en relación a la teoria rival de Lorentz. La ausencia de superioridad empírica está en la base del hecho de que el premio $\mathrm{N}$ obel no fue atribuído a Einstein por ella sino por el efecto fotoeléctrico. Feyerabend destaca que el programa de Lorentz parece haber sido abandonado más por el surgimiento de la M ecánica Cuántica que por el surgimiento de la TER.

\section{Referencia a aplicaciones de la T ER}

$\mathrm{N}$ uevamente, sin tomar partido en la discusión epistemológica en relación con el "contexto de aplicación" de una teoría, sería importante que los alumnos pudiesen analizar y reflexionar que la TER posibilita la interpretación y explicación de diversos fenómenos de la natural eza como el estudio de partículas subatómicas, fuentes de energía nuclear, etc., no estando, por lo tanto, restringida a cuestiones solamente de índole teórica. Este aspecto permite localizar la TER como una teoría física, o sea, vinculada con cuestiones empíricas y no como una tesis metafísica.

\section{- Repercusiones de la TER en distintos ámbitos}

LaTER, quizás como pocas otras teorías, ha trascendido el ámbito científico para influir, en algunos casos fuertemente, otros campos del saber, algunos hasta difícilmente imaginables por el alumno, como la filosofía (donde generó amplios debates), y el arte. Como se mencionó anteriormente, el incorporar estos aspectos contextualiza el conocimiento científico, mostrando que no es una actividad aislada y que puede modificar aspectos de la realidad insospechados. Esto es, que las grandes producciones del ámbito científico, no quedan circunscriptas al propio ámbito y pueden llegar a influenciar otros contextos. Es poco probable que estos vínculos puedan ser establecidos por los alumnos si el docente no estimula una reflexión en este sentido.

\section{Repercusión en el ámbito científico}

En el ambiente de los físicos la reacción ante la TER varió considerablemente de un país a otro. Es interesante destacar con los alumnos este aspecto, que ejemplifica que la ciencia no es ajena a las características sociales y culturales de un país, y estas pueden influir en forma positiva o negativa para la divulgación y apoyo de una teoría emergente. Tomando como referencia los países de mayor producción científica en ese momento, pasaremos a analizar, a modo de ejemplo, lo ocurrido en Alemania, Francia, Inglaterra y EE.UU. En el caso de Alemania, a diferencia de lo acontecido en los otros países mencionados, entre 1905 y 1941 la TER fue analizada por los físicos, aunque no había acuerdo de opiniones y no siempre fue interpretada en forma correcta. Para esto, fue relevante la figura de M ax Plank, quien siendo catedrático en Berlín y editor de Annalen der Physik, dio apoyo a la TER difundiéndola, así como su discípulo, von Laue, quien escribió el primer libro de la T ER en 1911. En contraposición, en Inglaterra se consideró a la T ER como un ataque al concepto de éter que era relevante para la Física británica en ese momento. Este hecho, además del creciente interés de los físicos británicos por la también recién nacida teoría atómica de Rutherford, influyó para que la TER pasase bastante inadvertida por años. En Francia y EE UU., por diversas razones, también tuvo inicialmente escasa repercusión. (Sanchez Ron, 1985; Villani and Arruda, 1998). En Francia, por ejemplo, Poincaré nunca mencionó la TER - se especula que fuese porque pensaba que era una parte mínima de su teoría (Villani, 1985, p. 38) - y en los Estados U nidos, no fue tomada en serio, por considerársela poco práctica 0 absurda. 


\section{Repercusiones en el ámbito de la Filosofía}

Existen una serie de problemas filosóficos que envuelven conceptos centrales modificados por la TER. Preguntas tales como: ¿D e qué tipo de objeto hablamos cuando hablamos del espacio y el tiempo? ¿Son ellos, o la combinación de ellos, una entidad sustancial, o se trata de conjuntos de relaciones entre cosas distintas a ellos mismos? 0 quizás, como pensaba Kant, son formas que la mente impone en un mundo no espacial y no temporal (Smart, 1975). Por eso, es interesante analizar las diversas respuestas que pueden darse a estas preguntas filosóficas desde las ideas científicas y observar las modificaciones que las mismas sufrieron con el aparecimiento de la TER.

Los conceptos de espacio y tiempo, predominantes en el paradigma M ecanicista, aparecen claramente plasmado en una de las principales obras $\mathrm{N}$ ewton: Ios Principia. En ella N ewton distingue el "tiempo absoluto, verdadero y matemático" del "tiempo relativo, aparente y común". Con respecto al espacio afirma que: "el espacio absoluto, por su propia naturaleza, sin relación a ninguna cosa externa, se mantiene semejante e inamovible" y además argumenta que: "el espacio relativo es al guna dimensión movible o medida de los espacios absolutos, que nuestros sentidos determinan por su posición respecto a los cuerpos, y que se toma, comúnmente, como espacio inamovible" (Smart, 1975, p. 159 y 160). A partir de la $T E R$, se asume la relatividad del espacio y el tiempo pero con un sentido diferente al asumido por la mecánica clásica, dado que no hay un sistema que pueda decirse que esté en "reposo absoluto", y por lo tanto el tiempo ya no es independiente del espacio como ocurría en la M ecánica Clásica.

Por eso, la TER revolucionó el pensamiento filosófico de la época, provocando debates y nuevas conceptualizaciones. Es interesante que los estudiantes puedan tener acceso a esta intima relación entre filosofía y ciencia que se remonta a los propios orígenes de la ciencia. D iscusiones como las de espacio y tiempo tienen unos veinticinco siglos de existencia y, sin embargo, hoy ninguna discusión entre filósofos que se ocupan de esos temas, puede ignorar la obra de Einstein (Boido et al., 1988)

\section{Repercusiones en el ámbito del arte}

Es interesante analizar las influencias de la ciencia en el arte, en particular en el caso de la Física sobre las concepciones de arte moderno, como las vanguardias desde 1910 en adelante. $\mathrm{No}$ son pocos los críticos o los historiadores del arte que intentan identificar influencias de TER y de la Relatividad $G$ eneral en al gunos rasgos característicos de los tantos "ismos" a los que dieron lugar las vanguardias entre 1910 y la Segunda Guerra Mundial. Si bien no todos tuvieron la misma trascendencia, algunos hicieron escuela, como por ejemplo el Cubismo, el Surrealismo o el Expresionismo. En algunos casos fue ron los críticos de arte quienes intentaron establecer las influencias (como en el caso del Cubismo) y en otros los propios artistas lo manifestaron públicamente. Es curioso que rastreando en las exposiciones de arte recientes pueden encontrase aún estas referencias (por ejemplo, las declaraciones del gran artista plástico $\mathrm{M}$ atta, con motivo de la presentación de su obra en Buenos Aires en 1998).

\footnotetext{
${ }^{9} \mathrm{Si}$ bien es cierto que para $\mathrm{N}$ ewton habría un espacio y un tiempo absolutos, ello no fue nunca aplicado en sus trabajos, pues era incontrolable. Por eso, dentro del programa mecanicista newtoniano siempre se trabajó con la relatividad galileana, para la cual, también, el tiempo es independiente del espacio.
} 


\section{- D iscusiones conceptuales}

En esta sección presentamos los aspectos conceptuales que consideramos deben ser enfatizados cuando se presenta a los alumnos las ideas centrales de la TER. Como ya indicamos en la Introducción varios trabajos indican las dificultades que los alumnos tienen para comprender sus conceptos fundamentales, lo que los lleva a interpretarlos desde matrices conceptuales clásicas o a la memorización de definiciones y fórmulas. Los resultados de las investigaciones apuntan también que no sólo los alumnos poseen errores relacionados con la TER, sino también los docentes. Los errores detectados en los docentes parecen relacionarse con una inadecuada formación en temas como laTER, pretendiendo encontrar, de la misma forma que los estudiantes, explicaciones de conceptos netamente relativistas en términos de la mecánica newtoniana (Aleman Berenger, 1997). Esto provoca una confusión de conceptos y una consecuente interpretación errónea de los fenómenos involucrados, interpretación ésta que posiblemente sea a posteriori transmitida a los estudiantes, estableciéndose de esta forma un círculo vicioso. Si a esto le sumamos una escasa discusión conceptual en los libros didácticos, dificilmente la TER pueda llegar a ser comprendida.

Esta comprensión conceptual, por otra parte, es fundamental en una visión contextualista de la enseñanza de la Física, visto que es imposible comprender el alcance y las consecuencias de una teoría científica sin una comprensión de lo que las ideas por ella expresadas representan y en que medida son nuevas y se separan de otras formas de entender los fenómenos físicos.

Teniendo en cuenta lo anterior, consideramos que sería importante que en los libros de texto se hiciera referencia explícita a los siguientes aspectos que detallamos a continuación.

\section{Profundización de la noción de movimiento relativo en el marco de la Mecánica Clásica}

En primer lugar, y antes mismo de presentar la TER, parecería ser fundamental una discusión profunda de las nociones de "sistema de referencia" y "movimiento relativo" en el sentido galileano, aunque esos temas ya hayan sido desarrollados antes del planteo relativista.

Respecto al concepto de "sistema de referencia" se ha encontrado que los alumnos poseen nociones alternativas al respecto, considerando que son un trozo de espacio vinculado a un cuerpo concreto, limitado por el tamaño y la forma de ese cuerpo, pudiendo contener otros cuerpos más pequeños en ese marco de referencia, donde un observador mira el fenómeno en cuestión (Panse et al., 1994). En este mismo trabajo se concluye además que la impresión general es que los alumnos de Física, aun los graduados, tienden a considerar el "sistema de referencia" como un truco decorativo, sin un fin explicativo y en general no demuestran poseer un entendimiento metaconceptual del concepto como herramienta que permite una formulación apropiada del principio físico de relatividad. Como continuación de esta investigación, Ramadas et al. (1996), analizaron cómo los estudiantes trabajaban con las transformaciones de tiempo, distancia, velocidad y energía entre sistemas de referencia, encontrando que los alumnos ante una situación concreta no tomaban en cuenta las leyes de transformación y se manejaban con sus ideas intuitivas de espacio y tiempo. Además rara vez utilizaban las leyes del principio de relatividad galileana a pesar que pueden simplificar bastante el problema. Confunden la invariancia de las leyes entre marcos de referencia en movimiento relativo con la invariancia del tiempo en determinado marco. Resultados seme jantes fueron encontrados por Pietrocola y Zylberstajn (1999) en estudiantes universitarios, 
algunos de los cuales, pertenecientes al último curso de profesorado en Física, ya habían visto contenidos de TER. Según estos autores, el Principio de Relatividad no es usado por los estudiantes en sus respuestas a situaciones donde el conocimiento y la aplicación de este principio son necesarias. M ás aún, las situaciones propuestas no fueron visualizadas como problemáticas, de forma que los estudiantes siquiera se sintieron en la necesidad de usar otras estructuras interpretativas que las de la mecánica clásica y del sentido común.

D adas las dificultades que los conceptos de "sistema de referencia" y "movimiento relativo" presentan, parece razonable entonces una discusión profunda de antes de abordar el estudio de la TER.

\section{D iscusión de conceptos contraintuitivos}

Existen numerosos estudios que muestran que los estudiantes utilizan los conceptos y resultados de la TER de acuerdo con un marco de referencia de pensamiento espontáneo y no en el sentido conceptual de la TER (Posner, et al. 1982; Villani y Pacca, 1987; G il y Solves, 1993; Arruda y Villani, 1996; Villani y Arruda, 1998). Así, por ejemplo, los conceptos de "contracción de las longitudes" y "dilatación del tiempo" son interpretados por los estudiantes como efectos aparentes resultantes de la percepción del observador, quienes recurren a conceptos de espacio y tiempo absolutos cuando tienen que resolver cuestiones simples relacionadas con la TER. M ás aún, los postulados de la TER resultan tan contraintuitivos para muchos estudiantes que, aun después de haber finalizado un curso de Física Contemporánea, se resisten a aceptarlos (Gil y Solbes, 1993; Toledo, Arriassecq y Santos, 1997). ${ }^{10}$ Por eso es fundamental incorporar esta discusión conceptual en los libros de texto y en el trabajo de aula.

\section{D iscusión del concepto de simultaneidad}

El concepto de simultaneidad suele resultar confuso para aquellos alumnos que no aceptan el principio de relatividad que exige la equivalencia de las leyes físicas en los sistemas inerciales. Así, los alumnos tienden a considerar más real la observación realizada por un observador ubicado en tierra que la realizada por uno en movimiento (Aleman Berenger y Pérez Selles, 2000). Por esta razón, sería importante profundizar que el concepto de simultaneidad entre sucesos espacialmente separados depende del sistema de referencia (Angotti et al., 1978).

\section{Esclarecimiento de paradojas}

A medida que la TER comenzó a ser discutida en el ámbito científico, surgieron apasionantes debates que fueron colocados habitualmente en la forma de paradojas. Sería interesante plantear en clase la discusión de alguna de ellas y explicar por qué, en realidad, no se constituyen en tales. Entre las más conocidas se encuentra la "paradoja de los gemelos" y la "paradoja de la contracción de la longitud" (M ook y Varguish, 1998).

Un buen ejemplo puede ser la paradoja de los gemelos, que suele interesar a los alumnos. En este caso se supone que dos astronautas, que tienen la particularidad de ser gemelos para poder comparar los efectos biológicos de la dilatación del tiempo en uno y en otro, se separan mediante un experimento, a una velocidad comparable con la de la luz. Se puede suponer que ambos se desplazan en naves espaciales con movimiento rectilíneo y

\footnotetext{
${ }^{10}$ Existen también trabajos que analizan los errores conceptuales de los docentes, aunque no se detallan aquí.
} 
uniforme alejándose uno del otro. D e esta manera, cada uno realiza sus cálculos (asumiendo que conocen laTER) y concluyen que es el otro quien permanece más joven. Sin embargo, no pueden "corroborarlo empíricamente" hasta estar uno frente al otro. Para esto, uno de los dos debe dejar de alejarse, dar la vuelta y ponerse a la par de la otra nave. Esto significa cambiar de velocidad, y aún cuando es posible despreciar la aceleración (haciendo que el viaje dure un tiempo lo suficientemente largo como que sea despreciable el tiempo que dura la aceleración) y asumir que el viaje de regreso también es realizado con movimiento rectilíneo uniforme, los mellizos estarán en dos sistemas inerciales distintos y esto es lo que hace que la situación sea asi métrica para ambos, quedando sin efecto la paradoja. Esta es una forma cualitativa de resolver la paradoja en el contexto de la TER. O tra opción es recurrir a una solución gráfica mediante los diagramas de espacio-tiempo de M inkowski. Si bien el álgebra involucrada en la formulación matemática que M inkowski realizó de la TER no sería comprensible para los alumnos del nivel medio de enseñanza, se podría intentar abordar la solución gráfica de ese planteo (ver, pór ejemplo, tópicos suplementarios A y B de Resnick, 1977 y los apéndices A y B de M ook y Varguish, 1998) que además permitiría retomar la discusión de los conceptos de sistema de referencia y simultaneidad.

Las paradojas (o los planteos que parecen serlo), por lo general, entusiasman a los alumnos y las discusiones que pueden generarse requieren la reconsideración de aspectos conceptuales centrales para la comprensión de la TER. Aquí pueden incluirse además discusiones acerca de realizaciones de cine que han tenido gran suceso y suelen entusiasmar a los adolescentes, analizando los errores conceptuales desde el punto de vista de la Física y particularmente de la TER. Ejemplos típicos son las tres versiones de "Volver al futuro", "Terminator I y II", "Viaje a las Estrellas" (en sus versiones de películas y serie televisiva) y "Experimento Filadelfia" (que hace alusión específica a un experimento de Relatividad"). En la mayoría de ellas, o bien se viola el principio de la velocidad de la luz como límite 0 bien se tergiversan las consecuencias de la TER y se acepta el hecho de viajar al pasado.

\section{Comentarios finales}

Con este trabajo, pretendemos realizar un aporte para la incorporación de contenidos más actuales en el currículo de Ciencias en el nivel medio y polimodal, dentro del marco de una postura contextualista en la enseñanza de la ciencia. C reemos que el estudio de casos históricos, como la T ER, situándolos en la propia época en la que se generó la teoría evitaría que los estudiantes se formen una imagen distorsionada de la metodología científica, de los científicos como "super genios", permitiendo visualizar el modo en que distintas ideas se van perfilando en concepciones más poderosas y se unen para formar entramados conceptuales, como lo son las teorías científicas. Este enfoque, además, hace posible valorar los aportes de diversos personajes históricos en la génesis de una concepción científica, aunque no hayan tenido éxito con posterioridad o el reconocimiento de sus pares en la época en que gestaron sus ideas.

$\mathrm{N}$ os parece importante resaltar una vez más que la presentación de la TER no puede quedar circunscripta al planteamiento histórico - epistemológico aislado de la discusión conceptual, que justamente le da sentido, o sea, las consideraciones históricas y epistemológicas en un vacío conceptual no tendrían sentido. Por eso, en esta presentacioón no pueden dejarse de lado los aportes de las investigaciones en relación con las dificultades de los estudiantes con los conceptos centrales de la TER.

Como indicamos en la introducción de este trabajo, una de las formas posibles de concretizar estas reflexiones es a través de los libros de texto que, como ya se mencionó 
anteriormente, son uno de los recursos más utilizados por los docentes y alumnos en el proceso de enseñanza - aprendizaje. Por esta razón nos parece que los ejes aquí propuestos podrían servir de base tanto para la elaboración de obras didácticas como para el análisis de los textos ya existentes; en este último aspecto estamos trabajando actualmente.

\section{Agradecimientos}

Agradecemos los valiosos (y desinteresados) aportes realizados por el Prof. Guillermo Boido y el Dr. Eduardo Flichman a una versión anterior de este trabajo como así también la crítica realizada por los réferis de la revista C iência \& Educação que sin lugar a dudas permitieron mejorar la calidad del presente trabajo.

\section{Bibliografía}

ALEM AN BEREN GER, R. A. Errores comunes sobre relatividad entre los profesores de enseñanza secundaria, Enseñanza de las Ciencias, v. 15,no 3, p. 301 - 307, 1997.

AN GOTTI, J. A., CALDAS, I. L., DELIZOIVOV, D., RÜDIN GER, E. y PERN AM BU $C O, M$. Teaching relativity with a different philosophy, American Journal of Physics, v. 46, № 12, p. 1258 - 1262, 1978.

ALEM AN BEREN GER, R. y PÉREZ SELLES, J. Enseñanza por cambio conceptual: de la Física Clásica a la Relatividad, Enseñanza de las Ciencias, v. 18,no 3, p. 463 - 471, 2000. ARRU D A, S. y VILLANI, A. Sobre as origens da relatividade especial: relações entre quanta e relatividade em 1905. Caderno Catarinense de Ensino de Física, v. 13, № 1, p. 32 - 47, 1996.

BO ID 0, G. N oticias del planeta Tierra: Galileo Galilè y la revolución científica. Buenos Aires: A-Z Editores, 1996.

BO ID O, G., FLICH M AN , E., YAGUE, J. y otros. Pensamiento Científico. Buenos Aires, CONICET, 1988.

CAPUAN 0 , Vicente et al. Física M oderna: ausente en la escuela media. M emorias de REF X, p. 2C - 03, 1997.

GIL PEREZ, D ., y SO LBES, J. The introduction of modern physics: overcoming a deformed vision of science, International Journal of Science Education, v. 15, № 5, p. 255 - 260, 1993.

H O D SO N , D. Philosophy of Science and Science Education. Journal of Philosophy of Education, v. 20, no 2, 1986.

HOLCOM B, D. Criterios para una actualización de los currículos de física en todos los niveles. M emoria del VI Conferencia Interamericana sobre Educación en la Física, 1997. H OLTON , G. Einstein, M ichelson and the Crucial experiment, en Thematic origins of scientific thought, Cambridge: H arvard University Press, p. 261 - 353, 1973.

H OLTON, G. Ensayos sobre el pensamiento científico en la época de Einstein. M adrid: Alianza Editorial, 1982.

ISLAS, S. y STIPCICH, S. Análisis de libros de texto en: Aportes para la Práctica D ocente en Ciencias de la $\mathrm{N}$ aturaleza en EGB 3. Tandil, Editado por la Universidad $\mathrm{N}$ acional del Centro de la Provincia de Buenos Aires, 2000. 
KRAGH , H . Introducción a la historia de la ciencia. Barcelona: Crítica, 1989. LAHERA, J. y FORTALEZA, A. Ideas previas de los alumnos sobre relatividad y resultados de un proceso de aprendizaje, Enseñanza de las Ciencias, N úmero extra, Tomo I, p. 227 y $228,1989$.

LIT W IN , E. 1997. Las configuraciones didácticas. Buenos. Aires: Paidós Educador, 1997. LO M BARDI, O. La pertinencia de la $H$ istoria en la enseñanza de ciencias: argumentos y contrargumentos. Enseñanza de las Ciencias, v. 15, № 3, p. 343 - 349, 1997. M AT TH EW S, M . R. H istoria, Filosofía y Enseñanza de las Ciencias: La aproximación actual. Enseñanza de las Ciencias, v. 12, no 2, p. 255 - 277, 1994.

M O OK, D. y VARGISH , T. La Relatividad: espacio, tiempo y movimiento. España: M c Graw Hill, 1998.

PAN SE, S., RAM AD AS, J. y KU M AR, A. Alternative conceptions in Galilean relativity: frames of reference, International Journal of Science Education, v. 16, № 1, p. $63-82$, 1994.

PIETRO COLA, M . y ZYLBERSZTAJN, A. The use of the principle of relativity in the interpretation of phenomena by undergraduate students. International Journal of Science Education. v. 21, ํㅜ 3, p. $261-276$.

POSNER, G. J., STIKE, K. A., HEW SO N, P. W. y GERTZO G, W. A. Accommodation of scientific conception: Toward a theory of conceptual change. Science Education, v. 66, no 2, p. 211 - 227, 1982.

RAM AD AS, J., BARVE, S. y KUM AR, A. Alternative conceptions in Galilean relativity: distance, time, energy and laws, International Journal of Science Education, v. 18, no 4, p. 463 - 477, 1996.

RESN ICK, R. Introducción a la Teoría Especial de la Relatividad. M éjico: Ed. Limusa, 1977.

SAN CHEZ RO N, J. M . El origen y desarrollo de la Relatividad. M adrid: Alianza, 1985. SM ART, J. Entre Ciencia y Filosofía. M adrid: Editorial Tecnos, 1975.

TO LED O, B., ARRIASSECQ , I. y SAN TO S, G.. "EI pasaje de la Física clásica a la Relativista desde la perspectiva del cambio conceptual". Enseñanza de las Ciencias, v. 15, no 1, p. 79 - 90, 1997.

TO U LM IN , S. La comprensión humana, Tomo 1: El uso colectivo y la evolución de conceptos. M adrid: Alianza Editorial, 1977.

VILLAN I, A. 0 confronto Lorentz-Einstein e suas interpretações (partes I, II, III, e IV). Revista de Ensino de Física, v. 3, № 1, 2, 3 y 4, 1981.

VILLAN I, A. A visão eletromagnética e a Relatividade (partes I e II), Revista de Ensino de Física, v. 7, № 1 y 2, 1985.

VILLANI, A. y PACCA, J. Students' spontaneous ideas about the speed of light, International Journal of Science Education, v. 9, no 1, p. 55 - 65, 1987.

VILLAN I, A. y PACCA, J. Spontaneous reasoning of graduate students. International Journal of Science Education, v. 12, no 5, p. 589 - 600, 1990.

VILLAN I, A. y ARRUDA, S. Special theory of relativity, conceptual change and history of science, Science \& Education, v. 7, p. 85 - 100, 1998. 
WILLIAM S, P. La teoría de la relatividad. M adrid: Ed. Alianza U niversidad, 1995.

Artigo recebido em 15 de outubro de 2001 e selecionado para publicação em 20 de março de 2002. 